\title{
MDC1 depletion promotes cisplatin induced cell death in cervical cancer cells
}

\author{
Neeru Singh ${ }^{2}$, Rashmi Bhakuni ${ }^{2}$, Dimple Chhabria ${ }^{2}$ and Sivapriya Kirubakaran ${ }^{1 *}$ (D)
}

\begin{abstract}
Objective: Cisplatin, the most common chemotherapeutic drug for the treatment of advanced stage cervical cancers has limitations in terms of drugs resistance observed in patients partly due to functional DNA damage repair (DDR) processes in the cell. Mediator of DNA damage checkpoint 1 (MDC1) is an important protein in the Ataxia telangiectasia mutated (ATM) mediated double stranded DNA break (DSB) repair pathway. In this regard, we investigated the effect of MDC1 change in expression on the cisplatin sensitivity in cervical cancer cells.

Results: Through modulation of MDC1 expression in the cervical cancer cell lines; Hela, SiHa and Caski, we found that all the three cell lines silenced for MDC1 exhibited higher sensitivity to cisplatin treatment with inefficiency in accumulation of $\mathrm{p}$ YH2AX, Ser 139 foci and increased accumulation of pChk2 Thr 68 at the damaged chromatin followed by enhanced apoptosis. Further, we observed the increased p53 Ser 15 phosphorylation in the MDC1 depleted cells. Our studies suggest that MDC1 expression could be a key determinant in cervical cancer prognosis and its depletion in combination with cisplatin has the potential to be explored for the sensitisation of chemo-resistant cervical cancer cells.
\end{abstract}

Keywords: Cisplatin, Cervical cancer, DNA damage repair, Ataxia telangiectasia mutated, MDC1, Apoptosis

\section{Introduction}

Cervical cancer is amongst the commonly diagnosed cancers in women, with a significant proportion of patients treated with cisplatin based chemo-radiotherapy (CRT) [1]. Genomic instability and high mutation rates exhibited in cervical cancers have been reported partly due to the persistent infection with human papillomavirus (HPV) and inactivation of important tumour suppressor genes [2, 3]. Few studies report that HPV proteins activate the ATM-Chk2 based DNA damage response pathway implicating its importance for viral genome amplification [4]. Thus, down-regulation of ATM pathway can prove to be an effective therapeutic approach towards blocking the spread of HPV infections. However,

\footnotetext{
*Correspondence: priyak@iitgn.ac.in

${ }^{1}$ Indian Institute of Technology Gandhinagar, Palaj Campus, Gandhinagar, Gujarat 382355, India

Full list of author information is available at the end of the article
}

the dejected outcomes including the lack of specific ATM kinase inhibitors [5] have stimulated research efforts to identify molecular characteristics specific to a certain tumour to predict their resistance (via the activation of DDR pathways) to CRT or recommend the utilization of novel targeted therapies [1] as cisplatin resistance remains one of the major concerns and causes of cervical cancer recurrences.

In this regard, MDC1, a mediator protein activated as part of ATM-ChK2 pathway is necessary for proper manifestation of the DDR response [6] and can be a limiting factor affecting prognosis of certain cancers [7]. MDC1 has-N-terminal fork head associated (FHA) domain, central PSTrich) domain and two C-terminal BRCT domains [8]. It regulates intra-S phase, G2/M checkpoints and actively participates in DSBs repair by both homologous recombination (HR) [9] and nonhomologous end joining (NHEJ) pathway [10]. At the site of damaged chromatin, ATM initiates a signalling cascade 
wherein a damaged chromatin is marked by $\gamma \mathrm{H} 2 \mathrm{AX}$, a Histone 2A variant phosphorylated at Serine 139 residue which is bound by phosphorylated MDC1 $[11,12]$. This interaction promotes the recruitment and retention of necessary DDR proteins at the damaged chromatin [1315] which supports positioning of additional ATM molecules resulting in amplification of DDR signalling along the chromatin length. MDC1 silencing in combination with radiation or chemotherapeutic agents presents a rational strategy for the treatment of ATM proficient cancers, which primarily depend on the ATM-Chk2 pathway activation for the repair of double strand DNA breaks. In the present study, we have shown MDC1 can explored as a therapeutic target for improving cervical cancer prognosis in combination with drugs like cisplatin.

\section{Main text}

\section{Materials and methods Cell culture}

Cervical cancer cell lines HeLa, SiHa and CasKi, were procured from National Centre for Cell Science, Pune. The cells were cultured in DMEM (Thermo Fisher Scientific, Waltham, MA, USA) with $10 \% \mathrm{FBS}, 100 \mathrm{U} / \mathrm{ml}$ penicillin, $100 \mu \mathrm{g} / \mathrm{ml}$ streptomycin, and $4 \mathrm{mM}$ L-glutamine and incubated at $37{ }^{\circ} \mathrm{C}$ in a CO2incubator. Cisplatin (P4394) was procured form Sigma Aldrich and diluted in $0.45 \% \mathrm{NaCl}$ saline solution.

\section{Antibodies}

p-H2AX Ser 139 mAb (2577S), p-p53 Ser15 mAb (9284S), p-Chk2 Thr68 mAb (2661) Anti-rabbit IgG HRP linked (7074), Anti-mouse IgG HRP linked secondary antibody (7076), Anti-IgG (H+L) F(ab')2 Alexa Fluor 488 (4412) and Anti-rabbit IgG $(\mathrm{H}+\mathrm{L}) \quad \mathrm{F}\left(\mathrm{ab}^{\prime}\right) 2$ Alexa Fluor 555 (Cat. 4413) were obtained from Cell Signalling Technology. MDC1 mouse mAb (1-50) (ab50003) from Abcam and anti human $\beta$-Actin (Cat No. SC47778HRP) was purchased form Santa Cruz Biotechnology.

\section{Stable cell line generation}

GIPZ lentiviral MDC1 shRNA (Dharmacon, Inc.) and pCDNA3 MDC1(a kind gift from Prof. Michel Goldberg, Hebrew University, Jerusalem) (Additional file 1: Figure S1 and Additional file 2: Figure S2) were used to generate the stable cervical cancer cell lines using Lipofectamine (Thermo fisher scientific). The stable cell lines were rigorously selected and maintained in $2.5 \mu \mathrm{g} / \mathrm{ml}$ puromycin and $400 \mu \mathrm{g} / \mathrm{ml} \mathrm{G418} \mathrm{for} \mathrm{MDC1} \mathrm{knockdown} \mathrm{and} \mathrm{MDC1}$ overexpression, respectively.

\section{Cell viability assay}

Cell viability assay was done using Cell Titer-Glo luminescent assay kit (Promega, USA) based on quantitation of the ATP. The cells were seeded at a count of 2000 cells per well in a 96 well plate and cisplatin treatment was given to the cells at different concentrations of 5,10 and $20 \mu \mathrm{M}$ for $72 \mathrm{~h}$ and luminescence reading (Envision, Perkin Elmer).

\section{Clonogenic survival assays}

Cells were treated with cisplatin $(10 \mu \mathrm{M})$ for $72 \mathrm{~h}$ after which they were trypsinized and seeded in $6 \mathrm{~cm}$ dishes in triplicate at a count of 1000 cells per plate. After 14 days of incubation the colonies were fixed with methanol and stained with crystal violet $(0.5 \%$ crystal violet in $20 \%$ Methanol, Sigma) and the colony numbers counted. The colonies with $\geq 50$ cell count as observed under a stereo microscope were considered for the analysis. Number of colonies derived from the untreated control cells was set as $100 \%$ (reference) for comparison. The surviving fraction was calculated by dividing the average number of visible colonies in treated versus untreated dishes.

\section{Western blotting}

Cells were seeded in $6 \mathrm{~cm}$ dishes $(0.8 \times 106 /$ dish $)$ and grown overnight. After treatment with cisplatin cells were lysed in ice-cold RIPA buffer (Sigma, R0278). The soluble fractions of cell lysate isolated by centrifugation at $13,000 \mathrm{rpm}$ for $10 \mathrm{~min}$ in a micro centrifuge at $4{ }^{\circ} \mathrm{C}$. and analysed by SDS-PAGE and western blotting (transfer onto PVDF membrane, Bio-Rad, USA). The membrane was blocked with $5 \%$ non-fat milk for $2 \mathrm{~h}$ at room temperature incubated with the primary antibody at $4{ }^{\circ} \mathrm{C}$ overnight with gentle shaking. Respective secondary antibodies were added the next day and blots were visualised using the Clarity Western ECL luminescent substrate (Cat No. 1705061) according to the manufacturer's instructions (Bio-Rad Laboratories). Beta-actin was used a loading control.

\section{Immunofluorescence assay}

Cells were seeded on poly L-lysine coated coverslips treated with cisplatin $(10 \mu \mathrm{M})$ for $2 \mathrm{~h}$ fixed with $4 \%$ paraformaldehyde for $15 \mathrm{~min}$, permeabilized with $0.3 \%$ Triton X-100/methanol solution for 10 min, blocked with bovine serum albumin/fetal bovine serum for $1 \mathrm{~h}$. Primary antibody incubation was performed overnight at $4{ }^{\circ} \mathrm{C}$. Cells were stained with TRITC labelled secondary antibody (Alexa Fluor 555 conjugate) for $\sim 2 \mathrm{~h}$ at room temperature. DAPI i.e., 4-6-diamidino-2-phenylindole dihydrochloride (Cat no. NC9524612; VECTASHIELD Antifade Mounting Medium, Fisher Scientific, USA) was used to stain the nuclei. 


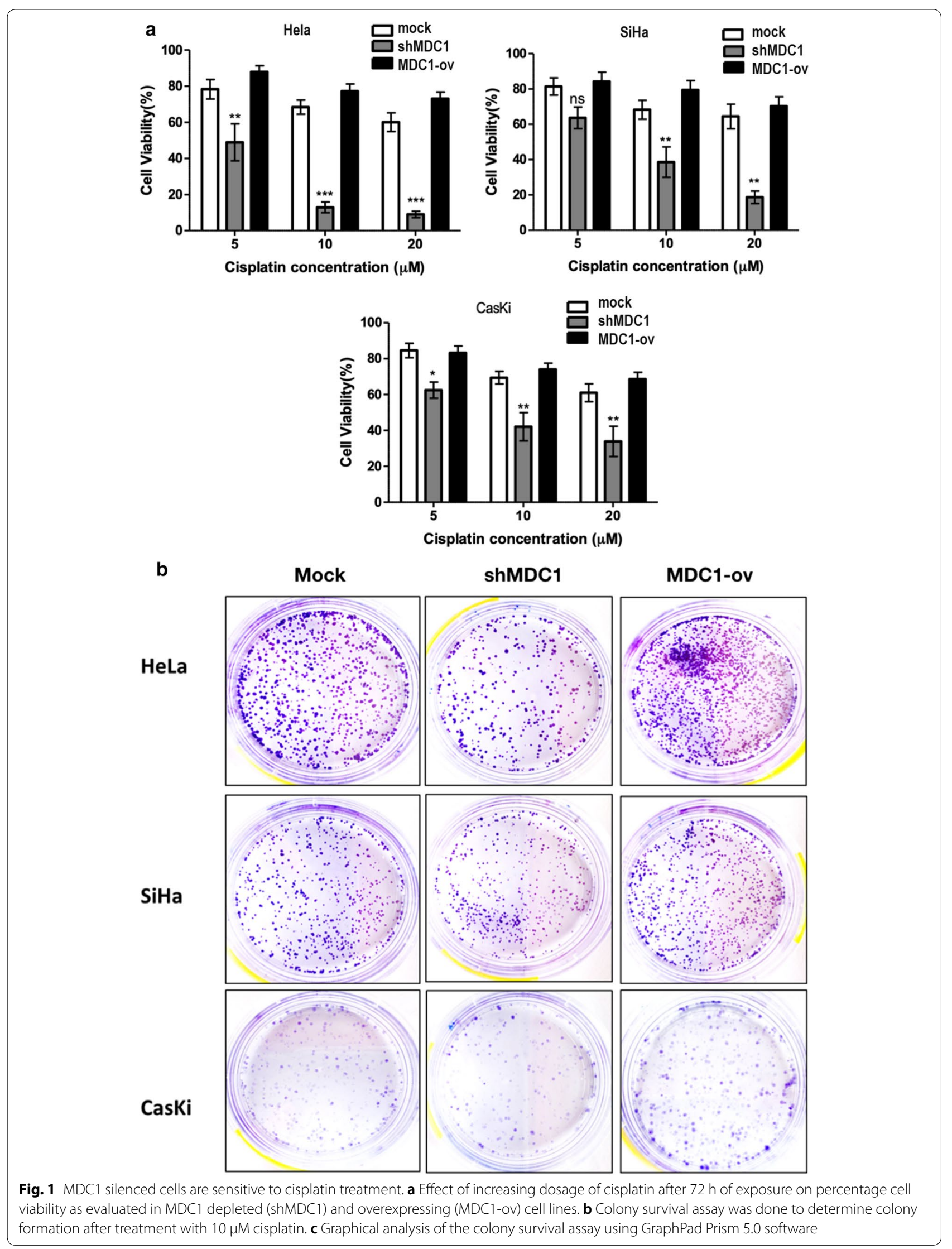



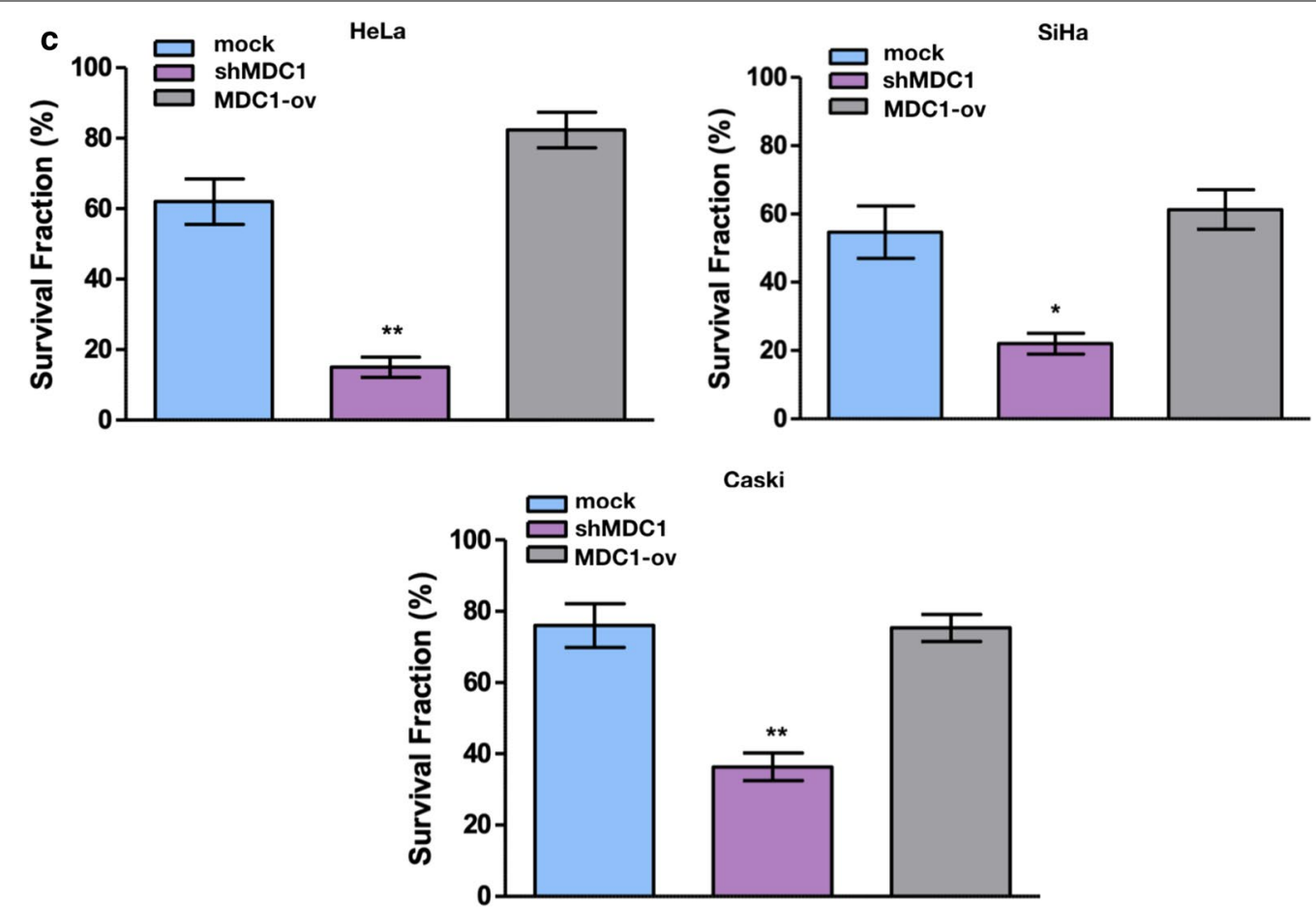

Fig. 1 continued

\section{Flow cytometry analysis of apoptosis}

Cells were seeded in $6 \mathrm{~cm}$ dishes, incubated for $24 \mathrm{~h}$ with $10 \mu \mathrm{M}$ Cisplatin when $80-90 \%$ confluent and stained with cyanine 3-conjugated annexin V (AnnexinV-Enzogold) and propidium iodide (PI) using the GFP certified Apoptosis/Necrosis Detection Kit (ENZ-51002, Enzo Biochem, Inc. New York, USA) as per the manufacturer's recommendation. Each sample was then subjected to analyses by flow cytometry (FCM) using S3e cell sorter (BIO-RAD, Hercules, CA, USA).

\section{Statistical analysis}

GraphPad Prism software (version 5.01) was used for statistical analysis, and $p<0.05$ was considered statistically significant (*p $<0.05,{ }^{* * *} p<0.01$, **** $\left.p<0.001\right)$. All the tests were either one-way or two-way analysis of variance (ANOVA) followed by multiple comparison post-test.

\section{Results}

\section{Stable cell line generation and effect of cisplatin on MDC1} expression modulated cervical cancer cells

Stable cervical cancer cell lines were generated (Additional file 3: Figure S3). We tested the sensitivity of the cell lines towards cisplatin at four different concentrations i.e. $5,10,15$ and $20 \mu \mathrm{M}$ for a duration of $72 \mathrm{~h}$ by CTGassay. Cisplatin exposure induced significant reduction in the cell viability in a dose dependent manner in MDC1 silenced cell lines (Fig. 1a).

Further, colony survival assay was performed after cisplatin $(10 \mu \mathrm{M})$ treatment). Considerably smaller number of surviving colonies were observed in MDC1 knocked down cell lines whereas MDC1 high expression rendered the cell lines insensitive to the drug treatment, evident as maximum number of colonies observed in these cell lines (Fig. 1b and c). The difference between control cells and MDC-ov cells was found to be statistically insignificant in all the three cervical cancer cell lines studied (Additional file 4: Figure S4).

\section{MDC1 promotes cisplatin induced $\mathrm{\gamma H} 2 \mathrm{AX}$ phosphorylation and foci formation and inhibits ChK2 accumulation}

MDC1 regulated $\gamma \mathrm{H} 2 \mathrm{AX}$ phosphorylation and interaction is a major signal for the recruitment of DDR proteins to the regions of damaged chromatin [15]. We performed immunofluorescence studies with our cell lines exposed to $2 \mathrm{~h}$ of cisplatin treatment and observed significant decline in the $\gamma$-H2AX foci formation in MDC1 knocked down cell lines with a concomitant increased accumulation of the same in MDC1 overexpressed cells. On the contrary, the Chk2 accumulation was noticed to be considerably high in MDC1 depleted cell lines supporting p53 stabilization and activation (Fig. 2). 


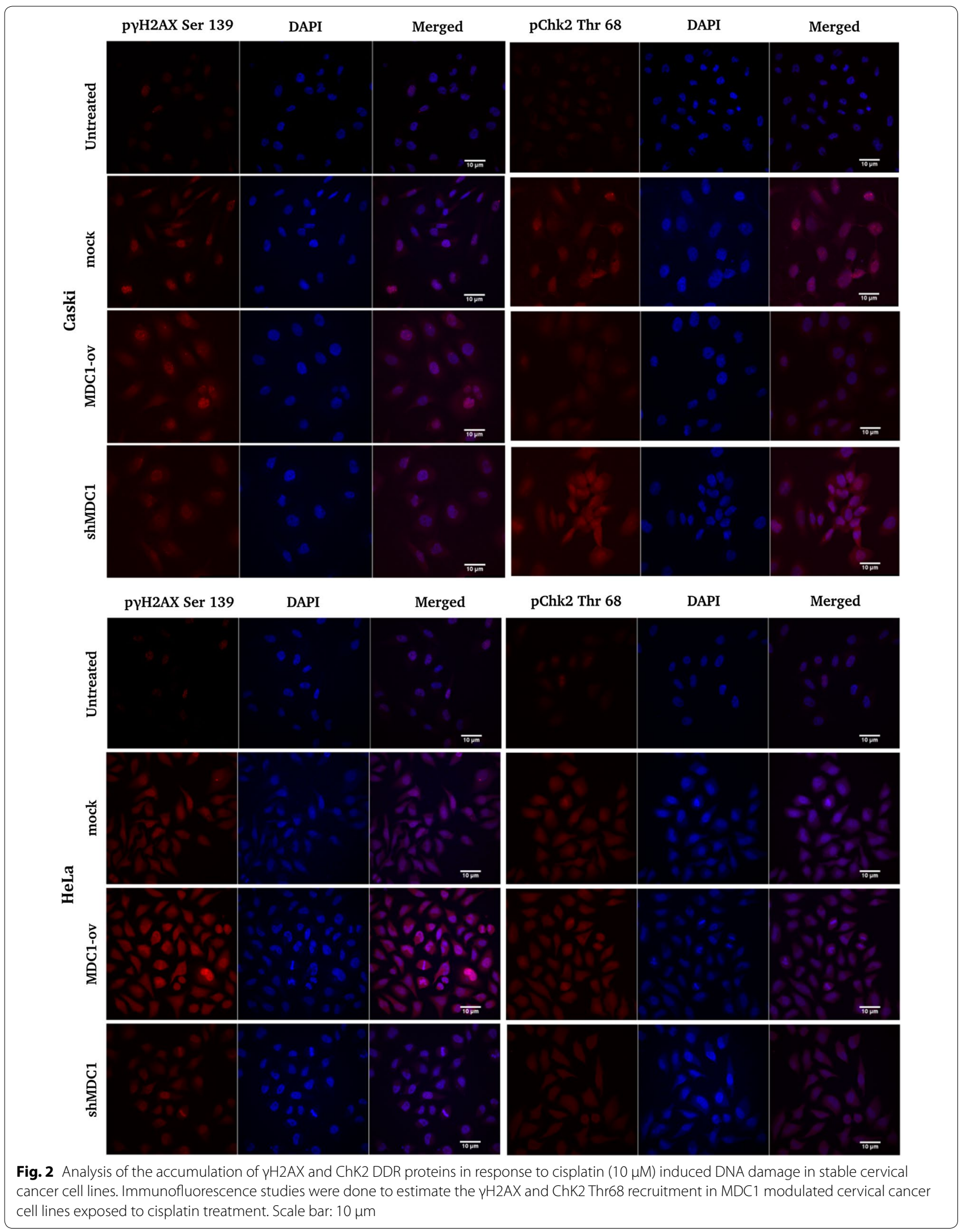




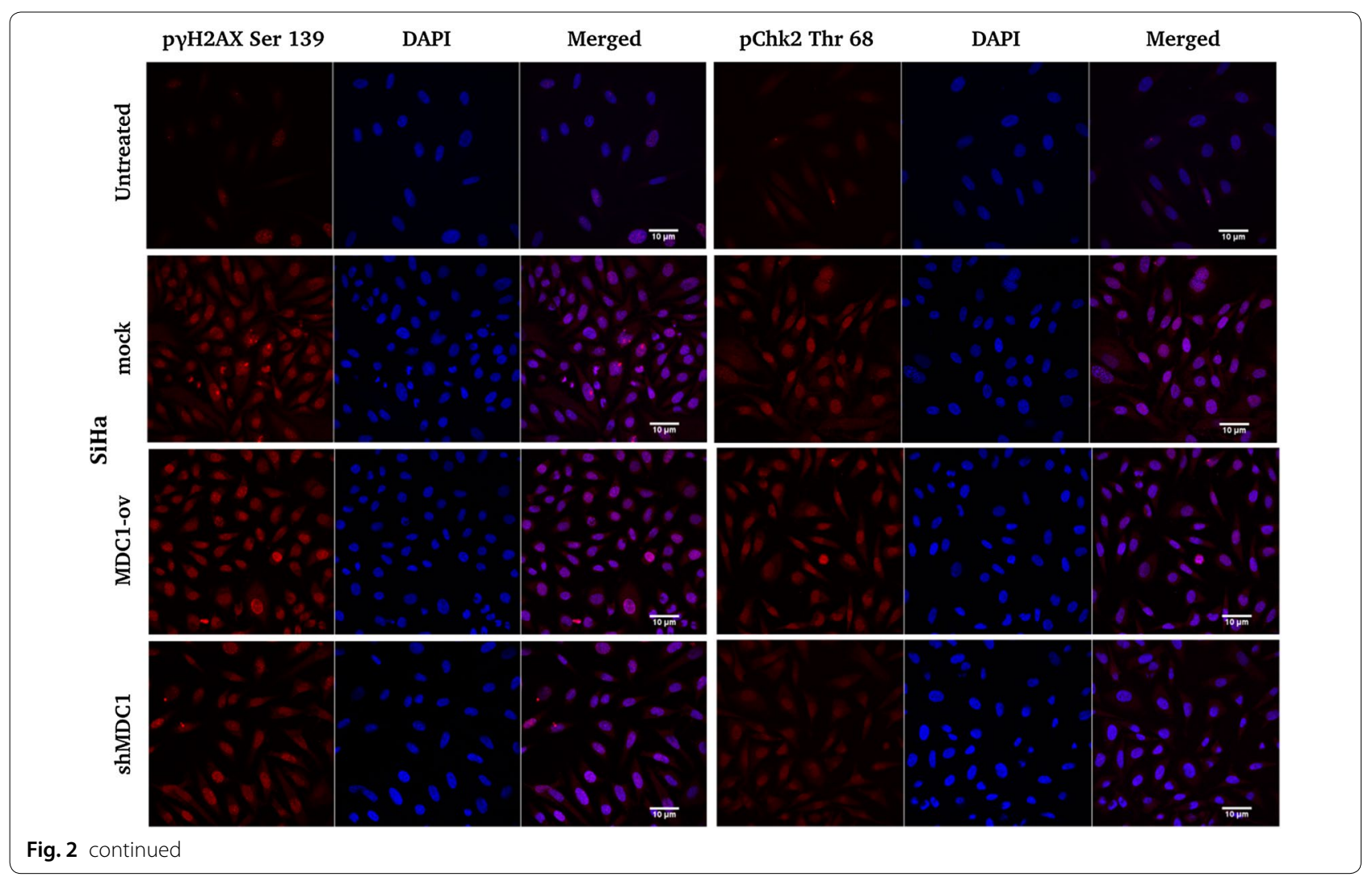

\section{Enhanced apoptosis in MDC1 knocked down cells upon cisplatin exposure followed by increased $p 53$ serine 15 phosphorylation}

To evaluate the apoptotic rate in the cell lines on cisplatin exposure, we performed annexin V and PI labelling after $24 \mathrm{~h}$ of treatment and monitored the cells by FCM. Our results showed high cisplatin sensitivity in combination with MDC1 depletion in all the three cervical cancer cell lines (Fig. 3a and b). Next, we analysed the phosphorylation status of p53 at Ser 15 residue by western blotting (Fig. 3c). The p53 function has been reported to be down regulated in case of HPV infected cervical cancer cells because of the overexpression of E6 and E7 oncogenic proteins [16, 17]. Simultaneously, Phosphorylation of p53 at Ser 15 is recognised as one of the central events in response to the ATM-Chk2 pathway induced double strand break repair [18]. Under conditions of sustained DNA damage expression of p53 Ser 15 phosphorylation was the most prominent in case of all the cells silenced for MDC1 expression as compared to the cells overexpressing MDC1. Also, p53 has been reported to escape E6 mediated degradation in cervical cancer cells after cisplatin treatment $[19,20]$. Although, the exact mechanism for the decrease in $\mathrm{p} 53$ phosphorylation in combination with MDC1 overexpression still remains elusive but on the basis of our results, it could be interpreted that down regulating MDC1 expression in cervical cancer cells favoured p53 mediated apoptosis in response to cisplatin treatment.

\section{Discussion}

Cervical carcinomas are often characterized by the abrogation of p53 tumor suppressor pathway mediated by HPV E6 and E7 oncogenes [21]. Additionally, HPV proteins are known to be responsible for activating the ATMChk2 pathway for aiding viral genome amplification [4]. Hence, regulation of ATM-Chk2 pathway can be a helpful approach towards addressing the spread of HPV infection in case of ATM proficient cells. Simultaneously, cisplatin has remained the first line of therapy for cervical cancer treatment and is reported to induce p53 mediated apoptosis [20]. The efficacy of cisplatin is limited by various factors including DNA repair mechanisms, which play a pivotal role in drug resistance [22]. Such factors together suggest the requirement for utilization of novel targeted therapies. We have shown that MDC1, a master regulator 


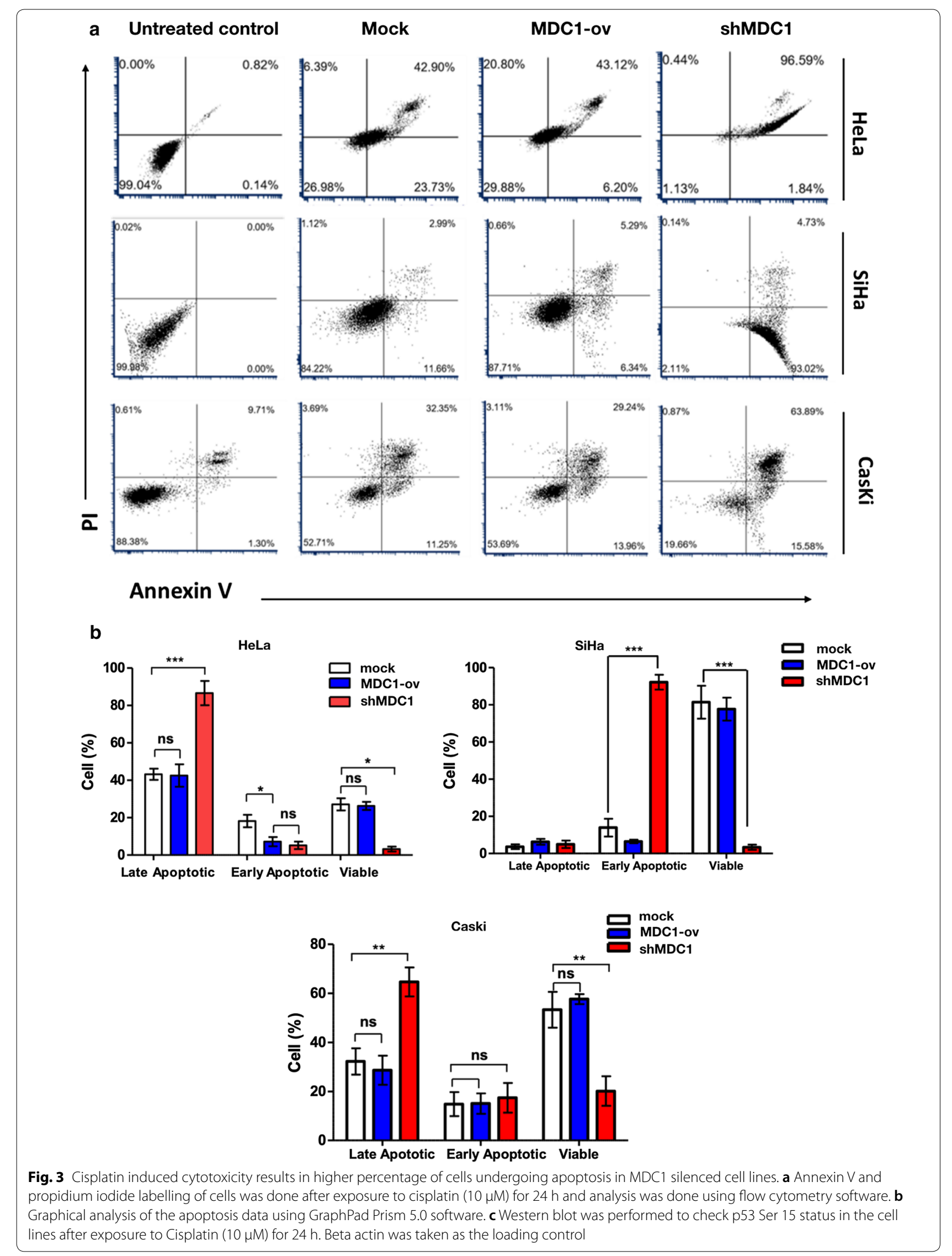




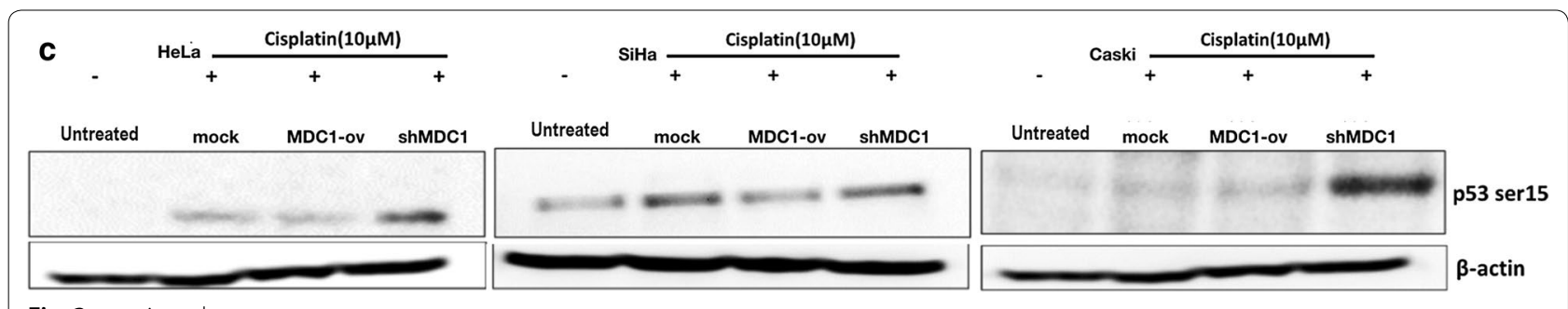

Fig. 3 continued

in the ATM-Chk2 pathway can be utilised for the sensitization of cervical cancer cells to chemotherapy. In the present study, we have evaluated the sensitivity to cisplatin in three high risk HPV positive cervical cancer cell lines with respect to the change in MDC1 expression. In our cisplatin treated cell lines, $\gamma$-H2AX accumulation was hindered in MDC1 silenced cells, resulting in defective foci formation in response to the DNA damage while the MDC1 overexpressing cells showed greater $\gamma-\mathrm{H} 2 \mathrm{AX}$ accumulation, indicating an efficient DDR and cell survival [23].

Our results noticeably indicate that MDC1 knock down increases sensitivity to cisplatin in the cervical cancer cell lines while its overexpression facilitates cisplatin insensitivity in the cells. Investigation of p53 Ser 15 status led us to conclude that MDC1 through its interaction with p53 determines cell survival or apoptosis in response to genotoxic stress. MDC1 overexpression leads to a lower level of apoptosis following damage induction, whereas its downregulation leads to higher levels of apoptosis. The phosphorylation of Serine 15 residue on p53 protein can be mediated by both ATM and ATR protein kinases and is quite important for $\mathrm{p} 53$ activation by promoting its phosphorylation on additional serine residues which are necessary for its proper stabilization [24, 25].

Furthermore, Chk2, a protein kinase that acts downstream of ATM kinase, plays an important role in increasing intracellular p53 levels in response to DNA damage and a decline in its expression causes a defect in p53-mediated apoptosis [26]. In general, Chk2 phosphorylated by ATM at threonine 68 residue actuates p53 phosphorylation on serine 20, which interferes with interaction between p53 and $\mathrm{Mdm} 2$ and hence, increases p53 stability by preventing its ubiquitination in response to DNA damage [27]. Our results indicate that MDC1 expression modulation does influence the phosphorylation of p53 protein and hence, modifies the apoptotic response to cisplatin treatment accordingly. As a result, the anti-apoptotic nature of MDC1 observed so far because of the inhibition of p53 apoptotic activity could be due to a direct interaction and binding between the two proteins which might block the transactivation domain of p53 from getting phosphorylated by ATM kinase and Chk2 upon DNA damage [28].

\section{Limitations}

Since cervical cancer cell lines are HPV positive monitoring E6 and E7 oncogene expression in our modified cell lines would have given greater insight as to increased cisplatin sensitivity in the MDC1 depleted cells. The investigation into p53 mediated apoptotic pathway becomes important factor in determining the fate of MDC1 silenced cells exposed to cisplatin. None the less the study clearly signifies that MDC1 in combination with cisplatin can prove to be an effective therapeutic approach for the treatment of cervical cancer patients.

\section{Supplementary information}

Supplementary information accompanies this paper at https://doi. org/10.1186/s13104-020-04996-5.

Additional file 1: Figure S1. GIPZ MDC1 shRNA vector map (Dharamcon, G.E, USA) used to develop MDC1 shRNA expressing cervical cancer cell lines. It expresses microRNA-adapted shRNA based on miR-30 for specific gene silencing with minimal cytotoxicity. The vector additionally has GFP gene expressing a green fluorescence protein and puromycin as the selection marker.

Additional file 2: Figure S2. Vector map of pCDNA3 MDC1 full length construct was received as a kind gift from Prof. Michel Goldberg, Hebrew University, Israel. The vector has G418 as the selection marker.

Additional file 3: Figure S3. Uncropped images of the HeLa, SiHa and Caski cell lines modified for MDC1 expression and assessed with MDC1 primary antibody and beta actin (as loading control).

Additional file 4: Figure S4. Quantification of immunofluorescence images generated for HeLa, SiHa and Caski cells lines subjected to staining with anti-pyH2AX Ser 139 antibody following $2 \mathrm{~h}$ of cisplatin treatment (for $\mathrm{p} y \mathrm{H} 2 \mathrm{AX}$ Ser 139 treated cells in Fig. 5). The images were quantified using ImageJ software and analysed for the corrected total cell fluorescence (CTCF). CTCF $=$ Integrated density - (Area of selected cell $\times$ Mean fluorescence of background readings).

\section{Abbreviations}

CRT: Chemoradiotherapy; MDC1: Mediator of DNA damage checkpoint 1; DDR: DNA damage repair; ATM: Ataxia telangiectasia mutated; Chk2: Checkpoint kinase 2; YH2AX: Gamma histone 2A X; HR: Homologous recombination; NHEJ: Non-Homologous End Joining.

\section{Acknowledgements}

Authors thank Prof. Michel Goldberg, Hebrew University, Israel for kindly providing the MDC1 full length construct. We also thank Dr. Vijay Thiruvenkatam and Dr. Virupakshi Soppina at IIT Gandhinagar for their kind support and valuable discussions. 


\section{Authors' contributions}

SK supervised the work. NS conceived the study and designed the experiments. NS, RB and DC performed the experiments. NS did statistical analysis, data interpretation and prepared the manuscript. SK and RB contributed in reviewing and editing the manuscript. All authors read and approved the final manuscript.

\section{Funding}

The authors thank Department of Science and Technology for awarding DST-women scientist grant for Neeru singh. The authors also thank SERB and DRDO for funding to Sivapriya Kirubakaran to carry out this work.

\section{Availability of data and materials}

Not applicable.

\section{Ethics approval and consent to participate}

Not applicable.

\section{Consent for publication}

Not applicable.

\section{Competing interests}

The authors declare that they have no competing interests.

\section{Author details}

${ }^{1}$ Indian Institute of Technology Gandhinagar, Palaj Campus, Gandhinagar, Gujarat 382355, India. ${ }^{2}$ Biological Engineering, Indian Institute of Technology Gandhinagar, Palaj, Gujarat 382355, India.

Received: 7 February 2020 Accepted: 3 March 2020 Published online: 11 March 2020

\section{References}

1. Ho CK, Kornaga EN, Klimowicz AC, Enwere EK, Dean M, Bebb GD, et al. Expression of DNA damage response proteins in cervical cancer patients treated with radical chemoradiotherapy. Gynecol Oncol. 2017;145:17684. https://doi.org/10.1016/j.ygyno.2016.12.025.

2. Ojesina Al, Lichtenstein L, Freeman SS, Pedamallu CS, Imaz-Rosshandler I, Pugh TJ, et al. Landscape of genomic alterations in cervical carcinomas. Nature. 2013;506:371. https://doi.org/10.1038/nature12881.

3. Werness BA, Levine AJ, Howley PM. Association of human papillomavirus types 16 and 18 E6 proteins with p53. Science. 1990;248:76-9. https://doi. org/10.1126/science.2157286.

4. Moody CA, Laimins LA. Human papillomaviruses activate the ATM DNA damage pathway for viral genome amplification upon differentiation. PLOS Pathog. 2009;5:e1000605. https://doi.org/10.1371/journ al.ppat.1000605.

5. Velic D, Couturier AM, Ferreira MT, Rodrigue A, Poirier GG, Fleury F, et al. DNA damage signalling and repair inhibitors: the long-sought-after Achilles' heel of cancer. Biomolecules. 2015. https://doi.org/10.3390/biom5 043204.

6. Jungmichel S, Stucki M. MDC1: the art of keeping things in focus. Chromosoma. 2010;119:337-49. https://doi.org/10.1007/s00412-010-0266-9.

7. Bartkova J, Hořejší Z, Sehested M, Nesland JM, Rajpert-De Meyts E, Skakkebæk NE, et al. DNA damage response mediators MDC1 and 53BP1: constitutive activation and aberrant loss in breast and lung cancer, but not in testicular germ cell tumours. Oncogene. 2007;26:741422. https://doi.org/10.1038/sj.onc.1210553.

8. Stewart GS, Wang B, Bignell CR, Taylor AMR, Elledge SJ. MDC1 is a mediator of the mammalian DNA damage checkpoint. Nature. 2003;421:961-6. https://doi.org/10.1038/nature01446.

9. Bakr A, Oing C, Köcher S, Borgmann K, Dornreiter I, Petersen C, et al. Involvement of ATM in homologous recombination after end resection and RAD51 nucleofilament formation. Nucleic Acids Res. 2015;43:3154-66. https://doi.org/10.1093/nar/gkv160.

10. Chang HHY, Pannunzio NR, Adachi N, Lieber MR. Non-homologous DNA end joining and alternative pathways to double-strand break repair. Nat Rev Mol Cell Biol. 2017;18:495. https://doi.org/10.1038/ nrm.2017.48
11. Rogakou EP, Boon C, Redon C, Bonner WM. Megabase chromatin domains involved in DNA double-strand breaks in vivo. J Cell Biol. 1999;146:905-16. https://doi.org/10.1083/jcb.146.5.905.

12. Stucki M, Clapperton JA, Mohammad D, Yaffe MB, Smerdon SJ, Jackson SP. MDC1 directly binds phosphorylated histone $\mathrm{H} 2 \mathrm{AX}$ to regulate cellular responses to dna double-strand breaks. Cell. 2005. https://doi. org/10.1016/j.cell.2005.09.038.

13. Lee J-H, Paull TT. ATM Activation by DNA double-strand breaks through the Mre11-Rad50-Nbs1 complex. Science. 2005;308:551-4. https://doi. org/10.1126/science.1108297.

14. Chapman JR, Jackson SP. Phospho-dependent interactions between NBS1 and MDC1 mediate chromatin retention of the MRN complex at sites of DNA damage. EMBO Rep. 2008;9:795-801. https://doi. org/10.1038/embor.2008.103.

15. Coster G, Goldberg M. The cellular response to DNA damage: a focus on MDC1 and its interacting proteins. Nucleus. 2010;1:166-78. https://doi. org/10.4161/nucl.1.2.11176.

16. Ahn WS, Han YJ, Bae SM, Kim T-H, Rho MS, Lee JM, et al. Differential suppression of human cervical cancer cell growth by adenovirus delivery of p53 in vitro: arrest phase of cell cycle is dependent on cell line. Jpn J Cancer Res. 2002;93:1012-9. https://doi.org/10.1111/j.1349-7006.2002. tb02478.x.

17. Koivusalo R, Krausz E, Ruotsalainen P, Helenius H, Hietanen S. Chemoradiation of cervical cancer cells: targeting human papillomavirus E6 and p53 leads to either augmented or attenuated apoptosis depending on the platinum carrier ligand. Cancer Res. 2002;62:7364-71.

18. Abraham RT. Cell cycle checkpoint signaling through the ATM an ATR kinases. Genes Dev. 2001;15:2177-96. https://doi.org/10.1101/gad.91440 1.dna.

19. Wsierska-Gdek J, Schloffer D, Kotala V, Horky M. Escape of p53 protein from E6-mediated degradation in HeLa cells after cisplatin therapy. Int J Cancer. 2002;101:128-36. https://doi.org/10.1002/ijc.10580.

20. Tan S, Hougardy BMT, Meersma GJ, Schaap B, de Vries EGE, van der Zee AGJ, et al. Human papilloma virus 16 E6 RNA interference enhances cisplatin and death receptor-mediated apoptosis in human cervical carcinoma cells. Mol Pharmacol. 2012;81:701-9. https://doi.org/10.1124/ mol.111.076539.

21. Magaldi TG, Almstead LL, Bellone S, Prevatt EG, Santin AD, DiMaio D. Primary human cervical carcinoma cells require human papillomavirus E6 and E7 expression for ongoing proliferation. Virology. 2012;422:114-24. https://doi.org/10.1016/j.virol.2011.10.012.

22. Khanna KK, Jackson SP. DNA double-strand breaks: signaling, repair and the cancer connection. Nat Genet. 2001;27:247-54. https://doi. org/10.1038/85798.

23. Stucki M, Jackson SP. $y H 2 A X$ and MDC1: anchoring the DNA-damageresponse machinery to broken chromosomes. DNA Repair. 2006;5:53443. https://doi.org/10.1016/j.dnarep.2006.01.012.

24. Tibbetts RS, Brumbaugh KM, Williams JM, Sarkaria JN, Cliby WA, Shieh SY, et al. A role for ATR in the DNA damage-induced phosphorylation of p53. Genes Dev. 1999;13:152-7. https://doi.org/10.1101/gad.13.2.152.

25. Loughery J, Cox M, Smith LM, Meek DW. Critical role for p53-serine 15 phosphorylation in stimulating transactivation at p53-responsive promoters. Nucleic Acids Res. 2014;42:7666-80. https://doi.org/10.1093/nar/ gku501.

26. Siliciano JD, Canman CE, Taya Y, Sakaguchi K, Appella E, Kastan MB. DNA damage induces phosphorylation of the amino terminus of p53. Genes Dev. 1997;11:3471-81. https://doi.org/10.1101/gad.11.24.3471.

27. Hirao A, Kong Y-Y, Matsuoka S, Wakeham A, Ruland J, Yoshida H, et al. DNA damage-induced activation of p53 by the checkpoint kinase Chk2. Science. 2000;287:1824-7. https://doi.org/10.1126/science.287.5459.1824.

28. Nakanishi M, Ozaki T, Yamamoto H, Hanamoto T, Kikuchi H. NFBD1/MDC1 associates with p53 and regulates its function at the crossroad between cell survival and death in response to DNA damage. J Biol Chem. 2007;282:22993-3004. https://doi.org/10.1074/jbc.M611412200.

\section{Publisher's Note}

Springer Nature remains neutral with regard to jurisdictional claims in published maps and institutional affiliations. 